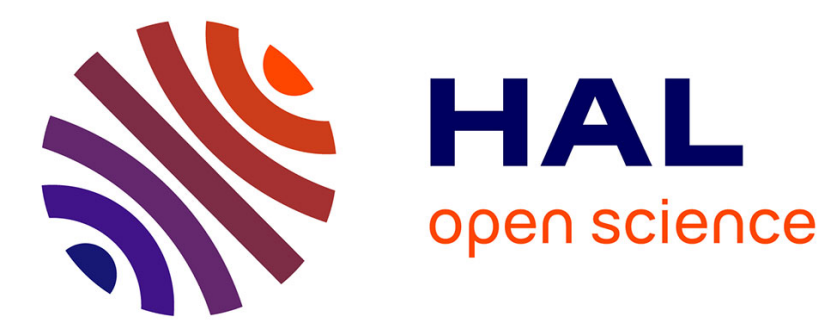

\title{
Nonlinear saturation of the large scale ow in a laboratory model of the quasibiennial oscillation
}

\author{
B. Semin, N. Garroum, F. Petrelis, S. Fauve
}

\section{To cite this version:}

B. Semin, N. Garroum, F. Petrelis, S. Fauve. Nonlinear saturation of the large scale ow in a laboratory model of the quasibiennial oscillation. Physical Review Letters, 2018, 121 (13), pp.134502. 10.1103/PhysRevLett.121.134502 . hal-01905951

\section{HAL Id: hal-01905951 \\ https://hal.sorbonne-universite.fr/hal-01905951}

Submitted on 26 Oct 2018

HAL is a multi-disciplinary open access archive for the deposit and dissemination of scientific research documents, whether they are published or not. The documents may come from teaching and research institutions in France or abroad, or from public or private research centers.
L'archive ouverte pluridisciplinaire HAL, est destinée au dépôt et à la diffusion de documents scientifiques de niveau recherche, publiés ou non, émanant des établissements d'enseignement et de recherche français ou étrangers, des laboratoires publics ou privés. 


\title{
Nonlinear saturation of the large scale flow in a laboratory model of the quasibiennial oscillation
}

\author{
B. Semin, N. Garroum, F. Pétrélis, S. Fauve \\ Laboratoire de Physique Statistique, École Normale Supérieure, PSL Research University; Université \\ Paris Diderot Sorbonne Paris-Cité; Sorbonne Universités UPMC Univ Paris 06; CNRS; \\ 24 rue Lhomond, 75005 Paris, France.
}

\begin{abstract}
The quasi-biennial oscillation (QBO) is the nearly periodic reversal of the large scale flow generated by internal waves in the equatorial stratosphere. Using a laboratory model experiment, we study the instability that generates the QBO and investigate its nonlinear regime. We report the first quantitative measurements of the nonlinearly saturated velocity of the flow. We show that the $\mathrm{QBO}$ is generated by a bifurcation that is either supercritical or subcritical depending on the dominant dissipative process. This is confirmed by a nonlinear analysis in the vicinity of the instability threshold.
\end{abstract}

\subsection{Ky, 47.55.Hd}

The quasi-biennial oscillation (QBO) is the nearly periodic reversal of the wind in the lower equatorial stratosphere. The period of the oscillation is 28 months on average, and is not locked to the yearly seasonal forcing. This wind has a significant influence on hurricanes in North America [1, and affects winter conditions in Europe [15].

This wind is known to be forced by atmospheric waves, in particular internal gravity waves that propagate in the stratosphere [10, 4, 23, 3, 14]. These waves generate a forcing at zero frequency and zero wave vector through the quadratic nonlinearities of the Navier-Stokes equation and therefore drive a flow at the largest scale of the system (for other experimental setups showing this phenomenon, see [9, 2]). This is an example of a large scale coherent field driven by small scale waves, other examples being acoustic streaming [12], mean flows generated by pattern-forming instabilities [5, 22 mean-field dynamo due to helical waves of velocity [16], to quote a few. This large scale flow displays reversals, as also observed for other types of large scale fields driven on a turbulent background [7] such as the magnetic field of the Earth or the Sun.

The mechanism responsible for the reversals was first identified by Lindzen and Holton [13] and a minimal model was proposed by Plumb [19]. It considers two internal waves propagating in opposite direction azimuthally and in the same direction vertically. One of these waves forces a mean flow eastward and the other one westward. The competition between these two waves results in a mean flow profile that changes sign at a given altitude. The position where the mean flow changes sign drifts towards the location from where the waves are emitted, which leads to a periodic reversal of the mean flow. This mechanism was confirmed experimentally [20, 18] and numerically [26]. The mean flow is oscillatory and its period is very large compared to the period of the wave, so that the phase of the mean flow oscillation is not locked to the one of the waves. The generation of a mean flow and its periodic reversals were observed experimentally but no quantitative measurement of the amplitude of the mean flow could be achieved and the type of the bifurcation remained unknown. Yoden and Holton [27] have shown that the reversals are generated by a supercritical Hopf bifurcation by numerically integrating Plumb's model when the dissipation of the mean flow is only due to bulk viscous 
effects. We are not aware of analytical predictions on the supercritical or subcritical nature of the bifurcation.

Yet, knowing the nature of the bifurcation of the instability which leads to the QBO is important to predict the possible scenarios if the forcing is modified, for example due to global warming. This is not only an academic exercise: an anomaly of the QBO has been recently measured [17]. In addition, determining the nature of the bifurcation is important for general circulation models in order to obtain the correct sensitivity to parameter changes.

In this letter, we investigate the nature of the bifurcation in a laboratory analogue of the QBO and we understand our results by analytically solving the model of Plumb and McEvan.

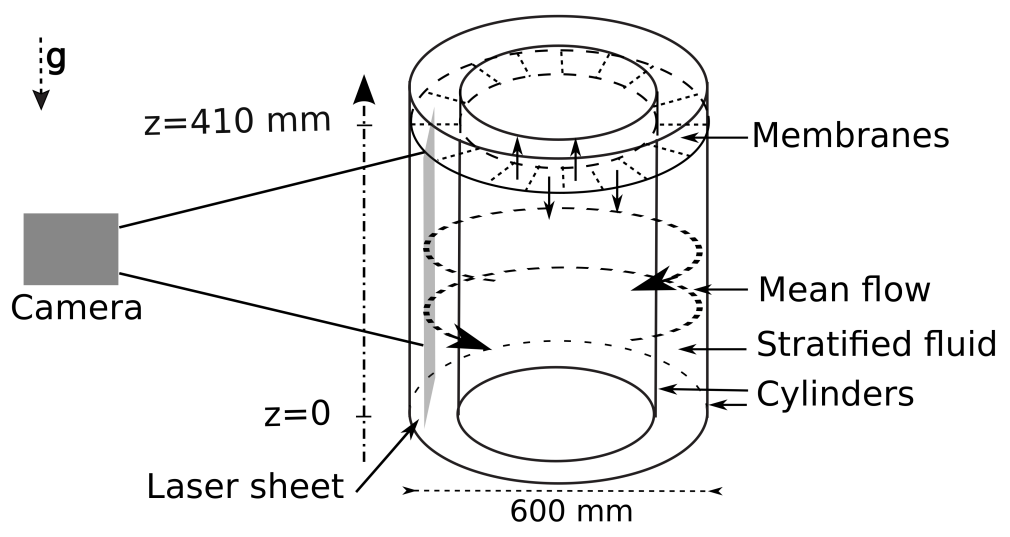

Figure 1: Schematic view of the experimental setup.

A schematic view of the experimental setup is shown in figure 1 and has been described in detail in [21]. It is made of two transparent concentric vertical cylinders. The outer diameter of the inner cylinder is $365 \mathrm{~mm}$, the inner diameter of the outer cylinder is $600 \mathrm{~mm}$, and the height is $H=410 \mathrm{~mm}$. The gap $h$ between these two cylinders is filled with a linearly density-stratified solution of $\mathrm{NaCl}$ or $\mathrm{MgCl}_{2}$ in water. The density profile is obtained from conductivity measurements. The stratification is measured by the Brunt-Väisälä frequency, $N=\sqrt{-\left(g / \rho_{0}\right) \mathrm{d} \rho_{0} / \mathrm{d} z}$, where $g$ is the acceleration of gravity, $\rho_{0}$ is the background density and $z$ is the vertical axis. The typical value of $N$ is $1.5-2.2 \mathrm{~Hz}$, the highest values been obtained using $\mathrm{MgCl}_{2}$.

The fluid motion is forced using 16 silicone membranes which are in contact with the top of the fluid and can move up and down in a nearly sinusoidal manner. Two neighboring membranes are driven by motors in opposition of phase so that the forcing is a standing wave whose azimuthal wavelength $\lambda_{x}=200 \mathrm{~mm}$ is twice the curvilinear distance between 2 motors. We note $M$ the amplitude of the motion of the membranes and we report here on measurements performed with a forcing period $T_{f}=15 \mathrm{~s}$.

The fluid velocity is measured using particle image velocimetry (PIV) [25]. To wit, the fluid is seeded with particles, whose density range is similar to the one of the fluid, so that particles can be found in the whole liquid (see supplemental material I and [21]). The wave (oscillation at the forcing angular frequency $\omega$ ) and the mean flow are deduced from these measurements. The mean flow is azimuthal and only depends on the height $z$, except close to the boundaries.

To investigate the bifurcation, the amplitude of the forcing is decreased step by step. We 
typically wait $8000 \mathrm{~s}$ for each value. The amplitude of oscillation of the mean flow is obtained by a fit using a sine function during the second half of each plateau of forcing.

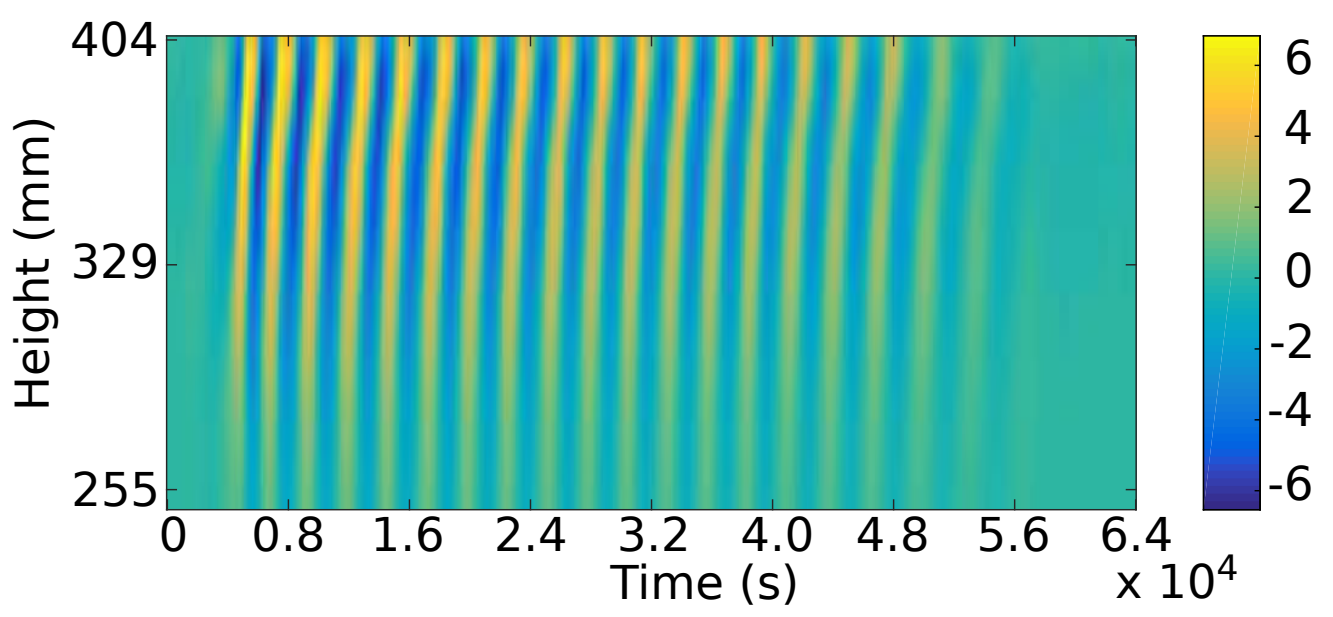

Figure 2: Space-time diagram of the mean flow. The color code indicates the velocity in $\mathrm{mm} \cdot \mathrm{s}^{-1} . \quad N=2.16 \mathrm{~Hz}\left(\mathrm{MgCl}_{2}\right)$ and $T_{f}=15 \mathrm{~s} . \quad M$ changes every $8000 \mathrm{~s}: M=$ $14.5,14,13.5,13,12.5,12,11.5,10.5,10 \mathrm{~mm}$.

We first describe the results obtained for high density gradient. For $N=2.16 \mathrm{~Hz}$, a spacetime diagram of the mean flow is displayed in figure 2. Since the first value of the forcing amplitude is high $(M=14.5 \mathrm{~mm})$, a mean flow is generated. During its growth, the mean flow is oscillating, and then reaches an oscillating steady state with a period of order $3000 \mathrm{~s}$ much larger than the period of the wave $\left(T_{f}=15 \mathrm{~s}\right)$.

We emphasize that we can keep the amplitude of oscillation constant on more than 20 periods whereas previous experiments did not report more than 2 periods. This results from a small pumping used to maintain the stratification beneath the membranes (see supplemental material II).

At fixed time, we observe that the flow changes direction at a given height. The point of zero mean flow moves upwards with time. This is in agreement with the behavior of the atmospheric QBO [1, where this point moves downwards: in the present experiment the waves are forced from the top and in the atmosphere the waves are forced below the layer of interest so that in both cases, the point of zero mean flow drifts toward the source of the waves.

When the amplitude of the forcing is decreased below a given value (here $M_{c}=11 \mathrm{~mm}$ ), the mean flow vanishes. This shows that there is a threshold in forcing amplitude below which the zero mean flow is stable.

To better visualize the time variation of the mean flow, we show it at a given height as as a function of time in figure 3a. Recordings at other heights are similar. We observe that the mean flow decreases smoothly when decreasing the forcing amplitude. The square of the amplitude $A$ of the mean flow (averaged in height) as a function of the forcing amplitude is shown in figure 4. The experimental data are well-fitted by a straight line which crosses the x-axis at a value in agreement with the threshold value $M_{c}$, thus showing that $A \propto \sqrt{M-M_{c}}$. The oscillation period varies with $M$ from $T \simeq 2200 \mathrm{~s}$ for $M=14.5 \mathrm{~mm}$ to $T \simeq 2900 \mathrm{~s}$ slightly above $M_{c}$. These two features characterize a supercritical Hopf bifurcation, i.e. the transition to an oscillatory regime with finite frequency at onset and continuously increasing amplitude of oscillation from zero. This transition to the QBO is observed when the Brunt-Väisälä 


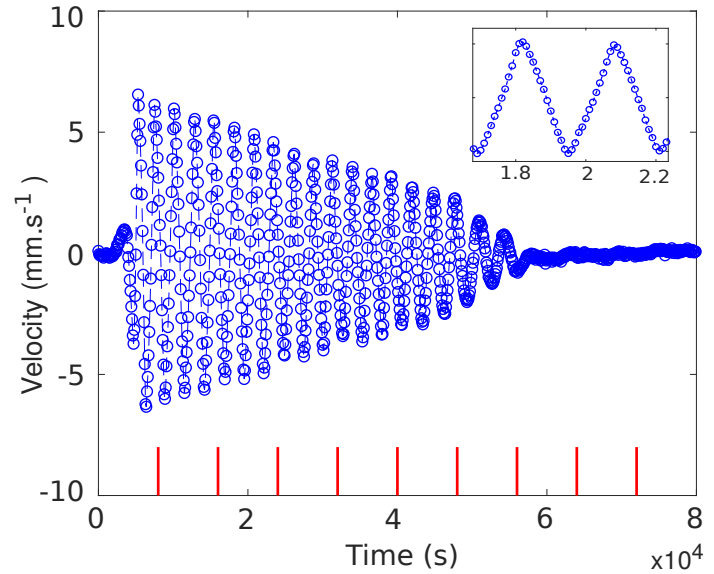

(a)

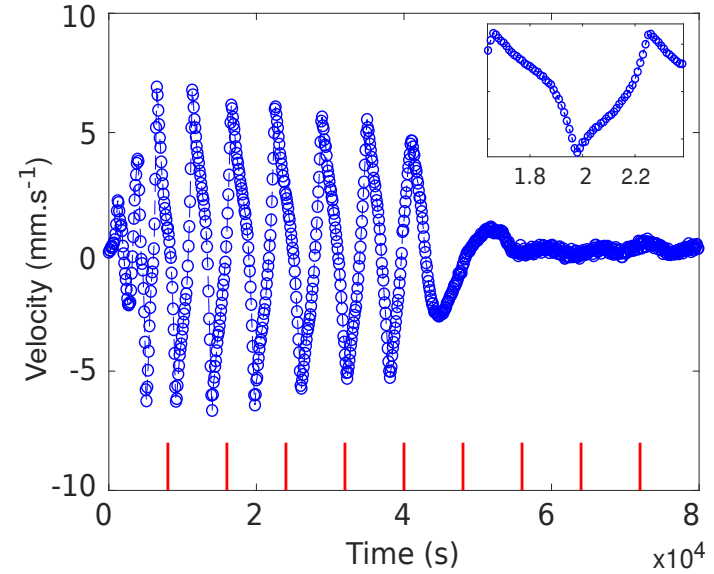

(b)

Figure 3: Mean flow as a function of time, for two different values of $N$, at height $h=376 \mathrm{~mm}$. $M$ changes every $8000 \mathrm{~s}$ (shown by vertical lines); $M=14.5,14,13.5,13,12.5,12,11.5,10.5,10$ mm. Inserts: details. (3a): $N=2.16 \mathrm{~Hz}\left(\mathrm{MgCl}_{2}\right) .(3 \mathrm{~b}): N=1.55 \mathrm{~Hz}(\mathrm{NaCl})$.

frequency is large enough, here $N=2.16 \mathrm{~Hz}$.

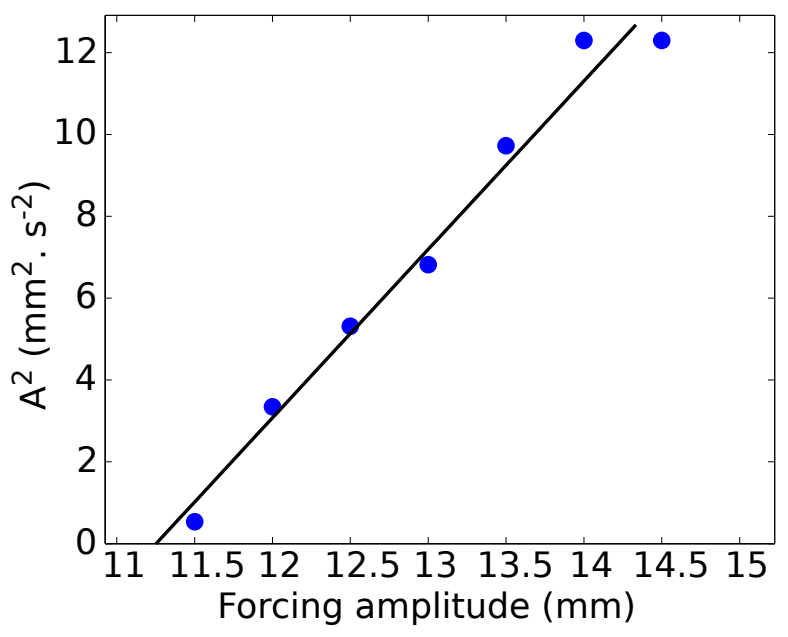

Figure 4: Square of the mean flow amplitude, $A^{2}$, as a function of $M(\bullet) . A$ is deduced from sine fits of the data of figure 2. Best fit (full line). $N=2.16 \mathrm{~Hz}\left(\mathrm{MgCl}_{2}\right)$.

Results at a lower value of the Brunt-Väisälä frequency are shown in figure $3 \mathrm{~b}$ for $N=$ $1.54 \mathrm{~Hz}$, where the forcing is changed in the same manner as in figure 3a. Compared to the results at large $N$, we observe the following. (i) the time series contains more harmonics (see insets of figure 3). (ii) The decrease of the mean flow with the forcing amplitude is much sharper. A plot similar to the one of figure 4 (not shown) displays a discontinuous jump of the amplitude to zero. (iii) In some experiments, the system remains in the zero mean flow state for long duration (we observed values up to $2 \times 10^{4} \mathrm{~s} \simeq 5.5 \mathrm{~h}$ for $M=13.5 \mathrm{~mm}$ ) and then suddenly transitions to an oscillation of large amplitude. All these observations show that at 
low value of $N$ the bifurcation is subcritical.

For the sake of completeness we add that the period of the oscillation is of order $4000 \mathrm{~s}$ and decreases with $M$. Earlier works used $\mathrm{NaCl}$ and water as a fluid and it is likely that the parameters used were in the regime of subcritical bifurcation. Subcriticality, together with the weakening of the stratification due to mixing, explain the difficulty in achieving reproducible and quantitative measurements of the mean flow.

We have shown experimentally that a change of $N$ changes the nature of the bifurcation. In the following, we will show using theoretical considerations that this change is due to a modification of the dominant dissipative term for the mean flow. We use the model proposed by Plumb and McEwan [20]. This model has been shown to describe quantitatively the generation of a mean flow by a single progressive internal wave in the setup used here (see Semin et al. [21]).

The equation for the dimensionless azimuthal mean flow $\tilde{u}(z, t)$ is

$$
\frac{\partial \tilde{u}}{\partial \tilde{t}}=-\sum_{n=1}^{2} \frac{\partial \tilde{F}_{n}}{\partial \tilde{z}}+\Lambda_{1} \frac{\partial^{2} \tilde{u}}{\partial \tilde{z}^{2}}-\Lambda_{2} \tilde{u}
$$

$\tilde{F}_{n}$ are the fluxes of impulsion per unit mass due to the waves

$$
\tilde{F}_{n}(\tilde{z}, \tilde{t})=\exp \left[-\int_{0}^{\tilde{z}} \frac{1}{\left(\tilde{u}-\tilde{c_{n}}\right)^{4}} \mathrm{~d} \tilde{z}^{\prime}\right]
$$

where $\tilde{c_{1}}=-\tilde{c_{2}}=1$ give the sign of the horizontal phase velocity of the wave. The amplitude of the flux at $\tilde{z}=0$ is 1 , which is a way to make the equation dimensionless.

The parameters of the model are related to the experimental parameters according to

$$
c=\frac{\omega}{k_{x}}, d=\left(\frac{N \gamma}{k_{x} c^{2}}+\frac{N^{3} \nu}{k_{x} c^{4}}\right)^{-1}, \tilde{z}=\frac{z}{d}, \tilde{u}=\frac{u}{c},
$$

where $\nu=10^{-6} \mathrm{~m}^{2} \cdot \mathrm{s}^{-1}$ is the kinematic viscosity of the fluid and $\gamma=10^{-3} \mathrm{~s}^{-1}$ is the damping rate due to friction at the cylinder walls. The dimensionless control parameters are

$$
\Lambda_{1}=\frac{\nu c}{F_{0} d}, \Lambda_{2}=\frac{\gamma c d}{F_{0}}
$$

where $F_{0}$ is the dimensional wave flux. It is proportional to $M^{2}$ and depends on properties of the membranes [20]. $1 / \Lambda_{i}$ are Reynolds numbers associated to different dissipative processes. We note that $\Lambda_{1} / \Lambda_{2}$ measures the ratio between viscous dissipation in the bulk and friction at the wall.

If we do not take into account the height $H$ and the gap $h$, six parameters, $N, \omega, c, F_{0}, \nu, \gamma$ are involved in the experiment with two units of length and time, therefore leading to four dimensionless numbers. Plumb's model is based on the smallness of $\omega / N$ and $c /(N d)$ and therefore involves two remaining dimensionless parameters $\Lambda_{1}$ and $\Lambda_{2}$. The height $H$ can be discarded because the damping length $d$ of the waves is smaller than $H$ and $h$ is partly taken into account by the value of the friction $\gamma$. We note from the values reported in Table 1 that both the experiment and the stratosphere involve fairly small values of $\omega / N$ and $c /(N d)$ and that the Reynolds number $1 / \Lambda_{1}$ is in the same range provided that a turbulent viscosity of the order of $0.1 \mathrm{~m}^{2} \cdot \mathrm{s}^{-1}$ is chosen for the stratosphere. This is not too surprising since the 
oscillation of the mean flow is not chaotic and therefore not far from its bifurcation threshold in both cases. Note however that the dissipation length $d$ involved in $\Lambda_{1}$ results from different dissipation mechanisms, because heat diffusivity and radiative damping are involved in the case of the stratosphere. However, in both cases, $c d / F_{0}$ gives a good order of magnitude for the period of the flow reversals [19].

\begin{tabular}{|r|r|r|r|r|r|r|}
\hline & $N$ & $d$ & $\omega / N$ & $c / N d$ & $1 / \Lambda_{1}$ & $c d / F_{0}$ \\
\hline Experiment & $2 \mathrm{~Hz}$ & $10 \mathrm{~cm}$ & 0.2 & 0.07 & $3-20$ & $2000 \mathrm{~s}$ \\
Stratosphere & $2 \times 10^{-2} \mathrm{~Hz}$ & $10 \mathrm{~km}$ & $10^{-4}$ & 0.15 & 30 & 1 year \\
\hline
\end{tabular}

We consider the model given by equations (1) and (2) in a semi-infinite domain $(\tilde{z} \in$ $[0,+\infty[)$. The boundary conditions are vanishing $\tilde{u}$ at infinity and at the forcing boundary $\tilde{z}=0$ (no-slip). The linear stability analysis shows that the mean flow is generated through a Hopf bifurcation with a normal mode given by integrals of Bessel functions. Weakly nonlinear analysis above threshold then gives the sign of the coefficient of the cubic term in the amplitude equation for the mean flow. This predicts the super-sub-criticality of the bifurcation. In addition, the form of the amplitude equation is not affected by a possible asymmetry between the counter propagating waves, because it is constrained by translational invariance in time. Details of these calculations are presented in supplemental material (III).

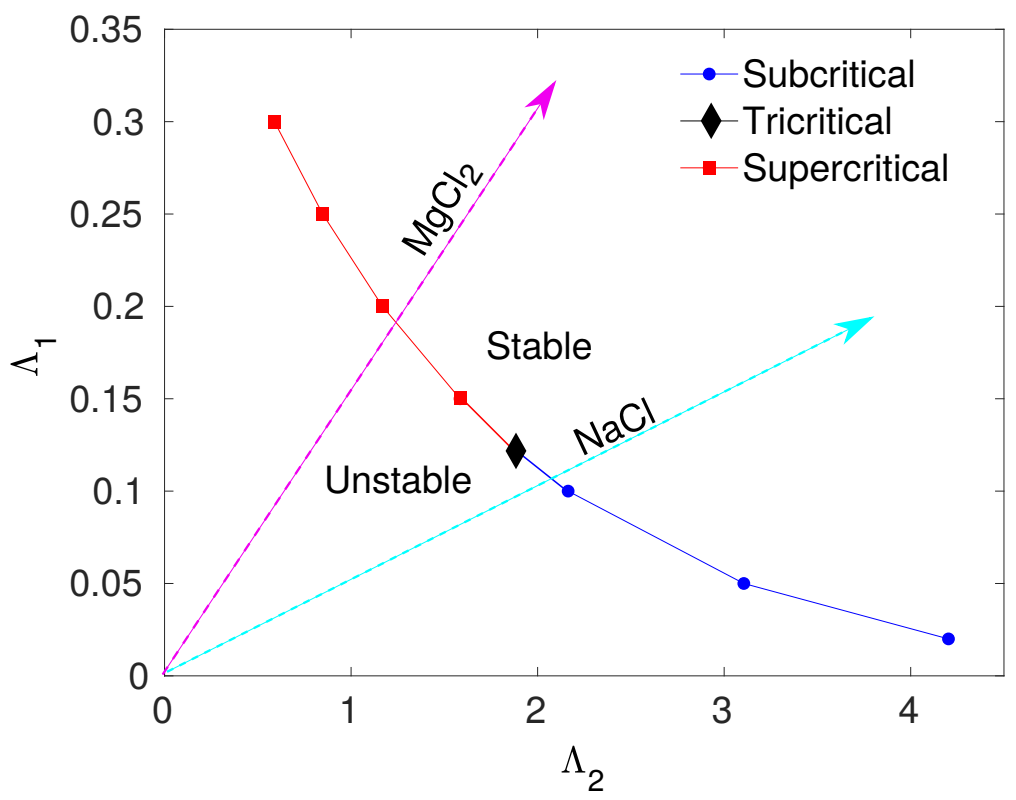

Figure 5: Parameter space displaying the transition between stable (no mean flow) and unstable regions. Instability threshold for large $N\left(\mathrm{MgCl}_{2}\right)$ and small $N(\mathrm{NaCl})$. Supercritical $(\boldsymbol{\square})$, subcritical $(\bullet)$ and tricritical $(\bullet)$ transitions.

The parameter space is shown in figure 5. The solution $\tilde{u}=0$ is linearly unstable at low values of $\Lambda_{1}$ and $\Lambda_{2}$, and linearly stable for large values. The bifurcation is supercritical 
for small values of $\Lambda_{2}$, and subcritical for large values. The result for small values of $\Lambda_{2}$ is consistent with the one of Yoden and Holton [27, who numerically observed a supercritical bifurcation when $\Lambda_{2}=0$. These two regimes are separated by a tricritical point located at $\Lambda_{1} \simeq 1.87$ and $\Lambda_{2} \simeq 0.12$. In the experiment, a change of $M$ leaves $\Lambda_{1} / \Lambda_{2}$ constant. The experimentally observed behaviors are in agreement with the results of the calculation.

In conclusion, we have shown using quantitative experiments and analytical calculations that the bifurcation in a QBO model is either supercritical or subcritical, depending on the dominant dissipative mechanism. A similar approach can be used to check that the transition to the atmospheric QBO described by Global Circulation Models also occurs through a Hopf bifurcation and to determine whether its super or sub-criticality also depends on the dominant dissipation mechanism. Finding the nature of the bifurcation can explain most qualitative features of the QBO. In particular, it shows why the phenomenon is not strongly affected by the asymmetry between waves traveling eastward and westward. In the astrophysical context, this approach could be applied to the QBO-like oscillations in atmospheres of planets such as Saturn [6] or Jupiter [11] or in the interior of stars [8, 24].

We gratefully acknowledge J. da Silva Quintas, C. Goncalves, E. Nicolau, C. Herrmann, and L. Bonnet for their technical support. This work has been supported by the Agence nationale de la recherche (Grants No. ANR-12-BS04-0005 and No. ANR-17-CE30-0004).

\section{References}

[1] M.P. Baldwin, L.J. Gray, T.J. Dunkerton, K. Hamilton, P.H. Haynes, J.R. Holton, M.J. Alexander, I. Hirota, T. Horinouchi, D.B.A. Jones, J.S. Kinnersley, C. Marquardt, K. Sato, and M. Takahashi. The quasi-biennial oscillation. Rev. Geophys., 39:179-229, 2001.

[2] G. Bordes, A. Venaille, S. Joubaud, P. Odier, and T. Dauxois. Experimental observation of a strong mean flow induced by internal gravity waves. Phys. Fluids, 24:086602, 2012.

[3] O. Bühler. Waves and Mean flows. Cambridge University Press, 2009.

[4] T. Dauxois, S. Joubaud, P. Odier, and T. Venaille. Instabilities of internal gravity wave beams. Annual Review of Fluid Mechanics, 50(1):131-156, 2018.

[5] A Davey, LM Hocking, and K Stewartson. Nonlinear evolution of 3-dimensional disturbances in plane poiseuille flow. J. Fluid Mech., 63:529-536, 1974.

[6] T. Fouchet, S. Guerlet, D. F. Strobel, A. A. Simon-Miller, B. Bézard, and F. M. Flasar. An equatorial oscillation in Saturn's middle atmosphere. Nature, 453:200-202, 2008.

[7] B. Gallet, J. Herault, C. Laroche, F. Petrelis, and S. Fauve. Reversals of a large-scale field generated over a turbulent background. GAFD, 106:468-492, 2012.

[8] E.-j. Kim and K. B. MacGregor. Gravity wave-driven flows in the solar tacholine. The Astrophysical Journal, 556:L117âL120, 2001.

[9] B. King, H. P. Zhang, and H. L. Swinney. Tidal flow over three-dimensional topography in a stratified fluid. Phys. Fluids, 21:116601, 2009. 
[10] Michael Le Bars, Daniel Lecoanet, Stéphane Perrard, Adolfo Ribeiro, Laetitia Rodet, Jonathan Aurnou, and Patrice Le Gal. Experimental study of internal wave generation by convection in water'. Fluid Dynamics Research, 47(4):045502, June 2015.

[11] X. Li and P.L. Read. A mechanistic model of the quasi-quadrennial oscillation in Jupiter's stratosphere. Planetary and Space Science, 36:637-669, 2000.

[12] J. Lighthill. Acoustic streaming. Journal of Sound and Vibrations, 61:391-418, 1978.

[13] R. S. Lindzen and J. R. Holton. A theory of the quasi-biennial oscillation. J. Atmos. Sci., 25:1095-1107, 1968.

[14] F. Lott and L. L. Guez. A stochastic parameterization of the gravity waves due to convection and its impact on the equatorial stratosphere. Journal of geophysical research: atmospheres, 118:8897-8909, 2013.

[15] A. G. Marshall and A. A. Scaife. Impact of the qbo on surface winter climate. Journal of Geophysical Research, 114:D18110, 2009.

[16] H. K. Moffatt. Magnetic Field Generation in Electrically Conducting Fluids. Cambridge University Press, 1978.

[17] P. A. Newman, L. Coy, S. Pawson, and L. R. Lait. The anomalous change in the QBO in 2015-2016. Geophys. Res. Lett, 43, 2016.

[18] N. Otobe, S. Sakai, S. Yoden, and M. Shiotani. Visualization and WKB analysis of the internal gravity wave in the QBO experiment. Nagare: Japan Soc. Fluid Mech., 17, 1998.

[19] R.A. Plumb. The interaction of two internal waves with the mean flow: implications for the theory of the quasi-biennial oscillations. J. Atmos. Sci., 34:1847-1858, 1977.

[20] R.A. Plumb and A.D. McEwan. The instability of a forced standing wave in a viscous stratified fluid : A laboratory analogue of the quasi-biennial oscillation. J. Atmos. Sci., 35:1827-1839, 1978.

[21] B. Semin, G. Facchini, F. Pétrélis, and S. Fauve. Generation of a mean flow by an internal wave. Physics of Fluids, 28:096601, 2016.

[22] ED Siggia and A Zippelius. Pattern selection in rayleigh-benard convection near threshold. Phys. Rev. Lett., 47(12):835-838, 1981.

[23] B. R. Sutherland. Internal Gravity Waves. Cambridge University Press, 2010.

[24] S. Talon, P. Kumar, and J.-P. Zahn. Angular momentum extraction by gravity waves in the sun. The Astrophysical Journal, 574:L175âL178, 2002.

[25] Z. J. Taylor, R. Gurka, G. A. Kopp, and A. Liberzon. Long-duration time-resolved PIV to study unsteady aerodynamics. IEEE Transactions on Instrumentation and Measurement, 59(12):3262-3269, 2010.

[26] N.P. Wedi and P.K. Smolarkiewicz. Direct numerical simulations of the Plumb-McEwan laboratory analog of the QBO. J. Atmos. Sci., 63:3226-3252, 2006. 
[27] S. Yoden and J. Holton. A new look at equatorial quasi-biennial oscillation models. J. Atmos. Sci., 45:2703-2717, 1988. 